\title{
Applying the Technology Acceptance Model to Evaluation of Recommender Systems
}

\author{
Marcelo G. Armentano, Ingrid Christensen, and Silvia Schiaffino
}

\begin{abstract}
In general, the study of recommender systems emphasizes the efficiency of techniques to provide accurate recommendations rather than factors influencing users' acceptance of the system; however, accuracy alone cannot account for users' satisfying experience. Bearing in mind this gap in the research, we apply the technology acceptance model (TAM) to evaluate user acceptance of a recommender system in the movies domain. Within the basic TAM model, we incorporate a new latent variable representing self-assessed user skills to use a recommender system. The experiment included 116 users who answered a satisfaction survey after using a movie recommender system. The results evince that perceived usefulness of the system has more impact than perceived ease of use to motivate acceptance of recommendations. Additionally, users' previous skills strongly influence perceived ease of use, which directly impacts on perceived usefulness of the system. These findings can assist developers of recommender systems in their attempt to maximize users' experience.
\end{abstract}

Index Terms-Recommender systems, evaluation, user acceptance, technology acceptance model.

\section{INTRODUCTION}

$\mathbf{T}$ HE popularization of the Web 2.0 has resulted in plethora of applications suggesting unlimited alternative items for users, which stresses the need for effective recommendations systems. In this context, recommender systems [1] are a popular solution, as they provide suggestions based on data about users' preferences, item attributes and relationships among users and items. Although the main objective has been placed on improving the algorithms to generate recommendations, it is now possible o understand that recommendation accuracy by itself is not enough to provide users with a satisfying experience. Therefore research has started to explore the factors that might have a direct impact on a user's acceptance of a recommendation technology, in order to maximize the popularity of a recommendation system; some of those factors are the user's satisfaction with the recommendations, suggested item attractiveness, accurate understanding of the user's preferences, intention to reuse the system, facility to evaluate candidate items, ease of use, capacity to learn and interact with the system. Swearingen and Sinha [2] were among the firsts to argue that the effectiveness of a recommender system depends on factors

Manuscript received on May 5, 2015, accepted for publication on June 5, 2015, published on June 15, 2015.

The authors are with the ISISTAN Research Institute (CONICET / UNICEN), Tandil, Argentina (e-mail: \{marcelo.armentano, ingrid.christensen, silvia.schiaffino\}@isistan.unicen.edu.ar). that go beyond the quality of the prediction algorithm. Several models attempted to address this problem, explaining and predicting the use of a system; nonetheless the Technology Acceptance Model (TAM) has been the one that has met with approval within the Information Systems community [3].

This paper aims at exploring potential user acceptance issues on a traditional recommender system, using the TAM. Within the basic TAM model, we incorporate a new latent variable representing self-assessed user skills to use a recommender system. We conducted an empirical user study using a movie recommender system as a testbed, as well as a questionnaire applicable to any recommender system in the entertainment domain (books, music, movies, etc.). The results evidence that the two main factors impacting on user acceptance are perceived usefulness and perceived ease of use, which is also affected by supposed skills in the use of recommender systems.

The remainder of this paper is organized as follows. Section 2 presents the Technology Acceptance Model used in our study. Section 3 presents some related work regarding the application of TAM to recommender systems. Section 4 describes the methodology used in our study and Section 5 presents the results obtained. Finally, Section 6 presents our conclusions.

\section{BACKGROUND}

Users' acceptance of a recommendation technology involves a set of variables regarding the users' experience in the use of the system that are related to the positive aspects of the interaction and to the fact of being captivated by a web application that leads to using it in a regular basis. User acceptance is a complex concept that goes far beyond having an attractive and easy-to-use user interface. It has been shown that two systems with identical user interface might be perceived differently by users if, for example, the underlying recommendation algorithm is changed [4].

So, what are the factors that influence the acceptance or rejection of an information technology? Davis [5] was among the firsts to study this question. First, he found that people will use an application if they believe it will help them to perform a given task better than when not using the application. Second, he found that even if users believe that a given application is useful, if the application is hard to use, then the perceived benefits of using the application are outweighed by the effort needed to use it. He call the first variable 
"perceived usefulness" and the second variable "perceived ease of use". With these findings Davis proposed the Technology Acceptance Model (TAM), which is an adaptation of the Theory of Reasoned Action (TRA) [6] to specifically deal with the prediction of the acceptability of an information system. The purpose of this model is to predict the acceptability of a tool and to identify the modifications that must be brought to the system in order to make it acceptable to users.

As shown in Figure 1, TAM suggests that Perceived Usefulness (PU) and Perceived Ease of Use (PEOU) determine an individual's intention to use a system with intention to use serving as a mediator of actual system use.

The Perceived usefulness is defined by Davis as "the degree to which a person believes that using a particular system would enhance his or her job performance". A system scoring high in perceived usefulness is then one for which a user believes in the existence of a positive user-performance relationship.

Perceived ease of use, in contrast, refers to "the degree to which a person believes that using a particular system would be free from effort". Effort is a limited resource that a person may allocate to the different activities he/she is performing. If we make all other factors invariable, a system perceived to be easier to use than another is more likely to be accepted by users

\section{Previous Work}

Some works have applied the TAM model to evaluate user acceptance in recommender systems, with different purposes. For example, in [7] a virtual community recommender recommends optimal virtual communities for an active user using behavioral factors suggested in TAM. Authors of this article include a filtering function based on the user's needs type, which makes the recommendation process more effective and efficient. In [8] the TAM model is used to evaluate the adoption of a recommender system in retail industry and banking sector.

In [9] the authors evaluate an existing personality-based recommender system using the technology acceptance model. They also consider that when recommending music other factors such as emotion and mood have to be considered. Then, in [10] a modified version of the technology acceptance model is applied to assess the customer's acceptance of individual personalized recommendations generated in an online shopping experience. Additionally, in [11] the authors present a framework questionnaire based on TAM, named as ResQue (Recommender systems' quality of user experience), which categorizes a set of questions into four dimensions: (1) perceived system qualities, (2) user's belief derived from these qualities, (3) user's subjective attitude, and (4) user's behavioral intentions.

In [12] the technology acceptance model and partial least squares regression are used to investigate learners' acceptance of a learning companion recommendation system (LCRS) in Facebook. They considered the usage of Facebook and the system design characteristics as external variables in
TAM. Moreover, in [13] the authors propose a framework to evaluate recommender systems from the user's perspective. This framework describes that user experience depends on the user's subjective perception about some objective aspects of the system, such as the recommendation approach applied or the user interface, together with personal and situational characteristics. Similarly, in [14] a travel information recommender system is evaluated. The study found that most travelers tend to acquire the recommendation from the Internet or word-of-mouth by friends and family. Therefore authors suggest that travel information websites should consider showing friends' travel information as an important issue. An extension of TAM, UTAUT [15], is studied in a recommendation system in the context of e-commerce in [16]. Specifically, the concept of trust on technological artifacts is adapted to the UTAUT model and both hedonic and utilitarian product characteristics were considered attempting to present a comprehensive range of recommender systems.

Finally, in [17] the authors present a detailed review of the state-of-the-art about user experience and user acceptance research in recommender systems.

\section{Methodology}

The experiment was conducted with an invitation to students and researchers from two universities in Argentina, in which we introduced the new movie recommender system, shown in Figure 2. In order to have a balanced study, invitations were sent to people in the area of Computer Sciences and people in other areas of study, such as Economics, Law, Business Administration and Finances. We asked participants to register in the recommender system website and to use it until the system recommended at least 20 interesting movies. Finally, we asked participants to answer an online survey, composed of 19 questions in a Likert-5 scale with 1 corresponding to "strongly disagree" and 5 corresponding to "strongly agree". The questions presented to participants, along with the associated TAM variable are detailed below:

- SKILLS_01: I believe I have the ability to use recommender systems to get useful recommendations.

- SKILLS_02: I believe I am able to identify my preferences regarding the products offered by the recommender system to get useful recommendations.

- SKILLS_03: I believe I have the ability to evaluate and use the recommendations of the recommender system to choose good movies to watch.

- PEOU_01: My interaction with the recommender system was clear and easy to understand.

- PEOU_02: I found the recommender system easy to use.

- PEOU_03: It was easy for me to learn how to use the recommender system.

- PU_01: I found the recommended movies attractive.

- PU_02: The recommended movies were adequate for my mood.

- PU_03: The recommended movies were tailored to my taste. 


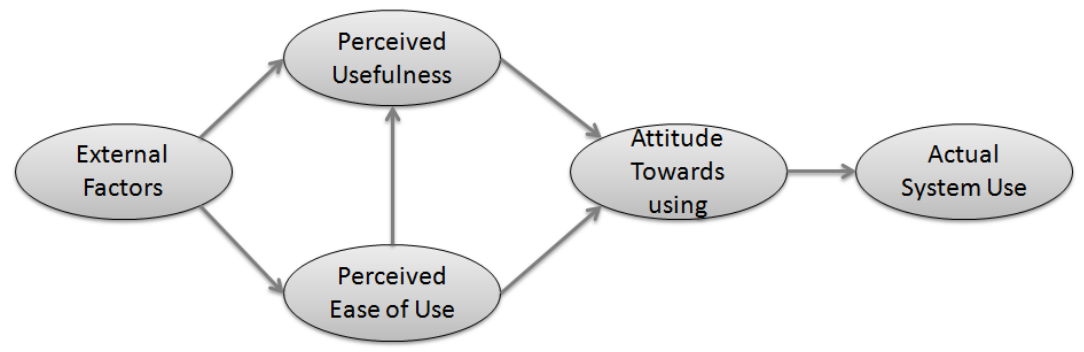

Fig. 1. Technology Acceptance Model

- PU_04: The recommended movies that I have already seen were movies I liked.

- PU_05: In general, I am satisfied with the recommended movies.

- PU_06: The recommended movies were as good as those that a friend would recommend.

- PU_07: The technology used by the recommender system is accurate.

- PU_08: The system understands my preferences regarding movies.

- ACCEPTANCE_01: I like the fact that the system learns about my preferences.

- ACCEPTANCE_02: I would use other recommender system in a different domain (songs, books, etc.).

- ACCEPTANCE_03: I want to own the recommended movies.

- ACCEPTANCE_04: I found the recommender system useful to find movies I liked and therefore I would use it again.

- ACCEPTANCE_05: I found the recommender system useful to find new movies that I would like to see and therefore I would use it again.

The experiment was open during December 2013, when we collected 116 cases. Table I shows some statistics of the participants of the experiment.

TABLE I

PARTICIPANTS STATISTICS

\begin{tabular}{cccc}
\hline Attribute & Variable & Rate & Amount \\
\hline \multirow{2}{*}{ Sex } & Male & $63.8 \%$ & 74 \\
& Female & $36.2 \%$ & 42 \\
\hline \multirow{3}{*}{ Age range } & $20-30$ & $76.7 \%$ & 89 \\
& $31-40$ & $14.7 \%$ & 17 \\
& $>40$ & $8.6 \%$ & 10 \\
\hline \multirow{4}{*}{ Area of Expertise } & Business & $32.8 \%$ & 38 \\
& Computer Sciences & $34.5 \%$ & 40 \\
& Economics & $17.2 \%$ & 20 \\
& Other & $2.6 \%$ & 3 \\
\hline
\end{tabular}

\section{EXPERIMENTS}

The variables of interest of TAM are often unobserved variables (latent variables). The "perceived usefulness", "perceived ease of use", "skills" and "acceptance" are variables that can not be directly observed, but that can be infered from some indicators, which are the answers to the questionary. The latent variables are modeled by specifying a measurement model and a structural model. The measurement model specifies the relationships between the observed indicators and the latent variables while the structural equation model specifies the relationships amongst the latent variables.

We performed an analysis that consisted in examining the reliability and validity of the measurement model (Section $\mathrm{V}-\mathrm{A}$ ) and examining the significance and prediction of path coefficients in the structural model (Section V-B).

\section{A. Measurement Model}

The first step was to determine the reliability and validity of the measurement model with item loadings, convergent validity, reliability of measure and discriminat validity.

Exploratory Factor Analysis (EFA) is a statistical approach for determining the correlation among the variables in a dataset. This type of analysis groups variables based on strong correlations, providing a factor structure. In Exploratory Factor Analysis there is no a priori theory about which items belong to which constructs. This means the EFA will be able to spot problematic questions in the experiment that do not fit well the latent variables they try to describe.

Principal Component Analysis (PCA) is a statistical procedure to perform EFA. It uses an orthogonal transformation to convert a set of observations of possibly correlated variables (questions in the questionary) into a set of values of linearly uncorrelated variables called principal components (TAM variables, in our case). Basically, PCA seeks a linear combination of variables such that maximum variance is extracted. This transformation assigns the largest possible variance (that is, accounts for as much of the variability in the data as possible) to the first principal component. Each succeeding component has the highest variance possible under the constraint that it is uncorrelated (orthogonal) with the preceding components. The principal components are orthogonal because they are the eigenvectors of the covariance matrix, which is symmetric.

In order to make the interpretation of the factors that are considered relevant, the first selection step is generally followed by a rotation of the factors that were retained. Two main types of rotation are used: orthogonal when the new 


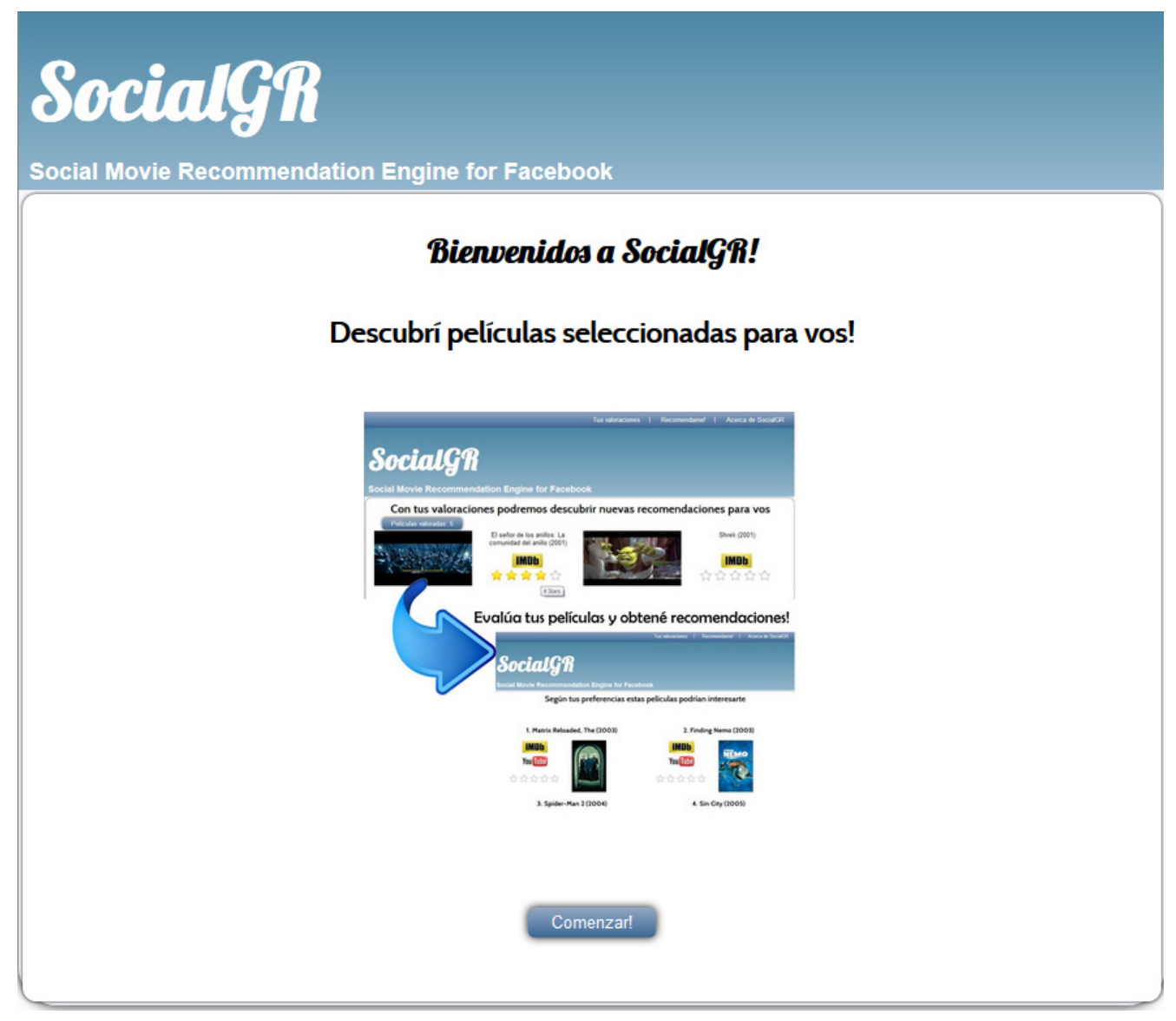

Fig. 2. Snapshot of the movie recommender system evaluated

axes are also orthogonal to each other, and oblique when the new axes are not required to be orthogonal to each other. Varimax [18] is the most popular rotation method. After a varimax rotation, each original variable tends to be associated with one or a small number of factors, and each factor represents only a small number of variables. In addition, the factors can often be interpreted from the opposition of few variables with positive loadings to few variables with negative loadings.

We performed Principal Component Analysis with Varimax rotation to extract factors from the questions asked to participants. Several tests were performed to check the suitability of the data for factor extraction:

- The Kaiser-Meyer-Olkin Measure (KMO) of sampling adequacy is a statistic that indicates the proportion of variance in the variables that might be caused by underlying factors. Values close to 1.0 generally indicate that a factor analysis may be useful with the data since patterns of correlations are relatively compact and then factor analysis should yield distinct and reliable factors. If the value is less than 0.50 , the results of the factor analysis probably will not be very useful. From our data, KMO measured 0.860 , which is indeed a very good index.
- Bartlett's test of sphericity tests the hypothesis that the correlation matrix is an identity matrix, which would indicate that the variables are unrelated and therefore unsuitable for structure detection. For factor analysis to work we need some relationships between variables and if the R-matrix were an identity matrix then all correlations coefficients would be zero. For our data, Barlett's test is highly significant $(\mathrm{p}<0.001)$ and therefore factor analysis is appropriate.

- Extraction communalities are estimates of the variance in each variable accounted for by the factors in the factor solution. Small values indicate variables that do not fit well with the factor solution, and should possibly be dropped from the analysis. The extraction communalities for our factors are acceptable, with the lowest 0.487 corresponding to ACCEPTANCE_01 (users like the fact that the system learns about their preferences). This means that $48.7 \%$ of the variance associated to ACCEPTANCE_01 is common, or shared, variance.

There are many indicators of the number of factors to retain from a EFA. The first approach is to consider the total variance explained by the retained factors. The total variance in the data is defined as the sum of the variances of the individual components. This quantity is simply the 
trace of the covariance matrix, since the diagonal elements of the latter contain the variances. On the other hand, the $\mathrm{K} 1$ method proposed by [19] is perhaps the best known and most utilized in practice. According to this rule, only the factors that have eigenvalues greater than one are retained for interpretation. Another popular approach is based on the Cattell's Scree test [20], which involves the visual exploration of a graphical representation of the eigenvalues. In this method, the eigenvalues are presented in descending order and linked with a line. Afterwards, the graph is examined to determine the point at which the last significant drop or break takes place-in other words, where the line levels off. The logic behind this method is that this point divides the important or major factors from the minor or trivial factors

Four factors in the initial solution resulted in eigenvalues greater than 1 . Table II shows the variance explained by each factor. Together, they account for $68.634 \%$ of the variability in the original variables. This suggests that, as expected for our research model, four latent influences are representative. This conclusion is supported by the scree plot in Figure 3

TABLE II

VARIANCE EXPLAINED BY EXTRACTED FACTORS

\begin{tabular}{cccl}
\hline Factor & Variance (\%) & Cumulative (\%) & Associated construct \\
\hline 1 & 38.53 & 38.53 & Perceived Usefulness \\
2 & 14.26 & 52.79 & Acceptance \\
3 & 8.87 & 61.66 & Perceived Ease of Use \\
4 & 6.97 & 68.63 & Skills \\
\hline
\end{tabular}

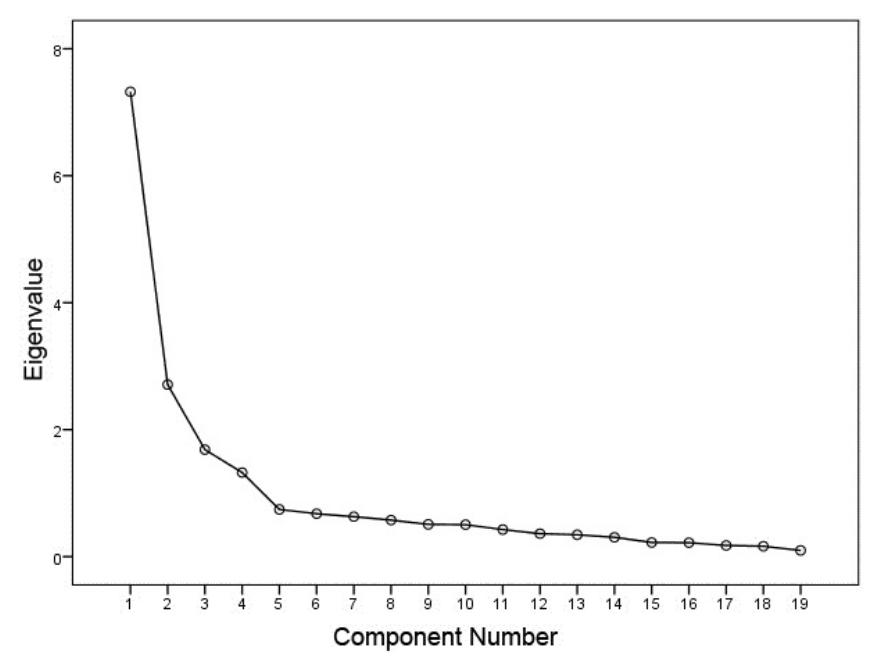

Fig. 3. Scree plot

Reliability refers to the consistency of the item-level errors within a single factor. A "reliable" set of variables will consistently load on the same factor. Cronbach's alpha is considered to be a measure of scale reliability or internal consistency. Cronbach's alpha can be written as a function of the number of test items and the average inter-correlation among the items. This metric measures how closely related a set of items are as a group. Table III shows the Cronbach's alpha coefficient on different factors. We can see that Cronbach-alpha is higher than 0.7 for all factors, indicating that the reliability of data can be considered to be sufficient.

TABLE III

CRONBACH'S ALPHA FOR DIFFERENT FACTORS

\begin{tabular}{lc}
\hline Factor & Cronbach's alpha \\
\hline Skills & 0.789 \\
PEOU & 0.836 \\
PU & 0.924 \\
Acceptance & 0.827 \\
TOTAL & 0.897 \\
\hline
\end{tabular}

Convergent validity means that the variables within a single factor are highly correlated. This is evident by the factor loadings. The factors extracted from the data demonstrate sufficient convergent validity, as their loadings were all above the recommended minimum threshold of 0.55 for samples size of 100 [21]. On the other hand, discriminant validity refers to the extent to which factors are distinct and uncorrelated. By examining the component matrix (Table IV) we can see that variables load significantly only on one factor, demonstrating sufficient discriminant validity.

TABLE IV

ROTATED COMPONENT MATRIX. EXTRACTION METHOD: PRINCIPAL COMPONENTS, ROTATION METHOD: VARIMAX WITH KAISER NORMALIZATION. ROTATION CONVERGED IN 6 ITERATIONS.

\begin{tabular}{lcccc}
\hline & \multicolumn{3}{c}{ Component } \\
\cline { 2 - 5 } & PU & Accept. & PEOU & Skills \\
\hline PU_03 & .902 & & & \\
PU_05 & .847 & & & \\
PU_01 & .812 & & & \\
PU_02 & .805 & & & \\
PU_04 & .763 & & & \\
PU_06 & .692 & & & \\
PU_08 & .653 & & & \\
PU_07 & .650 & & & \\
ACCEPTANCE_04 & & .781 & & \\
ACCEPTANCE_02 & & .739 & & \\
ACCEPTANCE_05 & & .737 & & \\
ACCEPTANCE_03 & & .643 & & \\
ACCEPTANCE_01 & & .633 & .909 & \\
PEOU_02 & & & .839 & \\
PEOU_03 & & & .729 & \\
PEOU_01 & & & & .844 \\
SKILLS_01 & & & & .806 \\
SKILLS_02 & & & & \\
SKILLS_03 & & & & \\
\hline
\end{tabular}

\section{B. Structural Model}

The next step after exploratory factor analysis is to confirm the factor structure we extracted. The objective of confirmatory factor analysis (CFA) is to test whether the data fit our research model. Model fit refers to how well our proposed model (in this case, the model of the factor structure) accounts for the correlations between variables in the questionary. If we are accounting for all the major correlations inherent in the answers to the questionary (with regards to the variables in TAM), then we will have good fit. Otherwhise, there is a 
significant "discrepancy" between the correlations proposed and the correlations observed, and thus we have poor model fit.

Regression Evaluation for the constructs was performed using AMOS. Figure 4 shows the stardardized estimates regarding each question, all estimates are significant at $\mathrm{p}<0.001$ level.

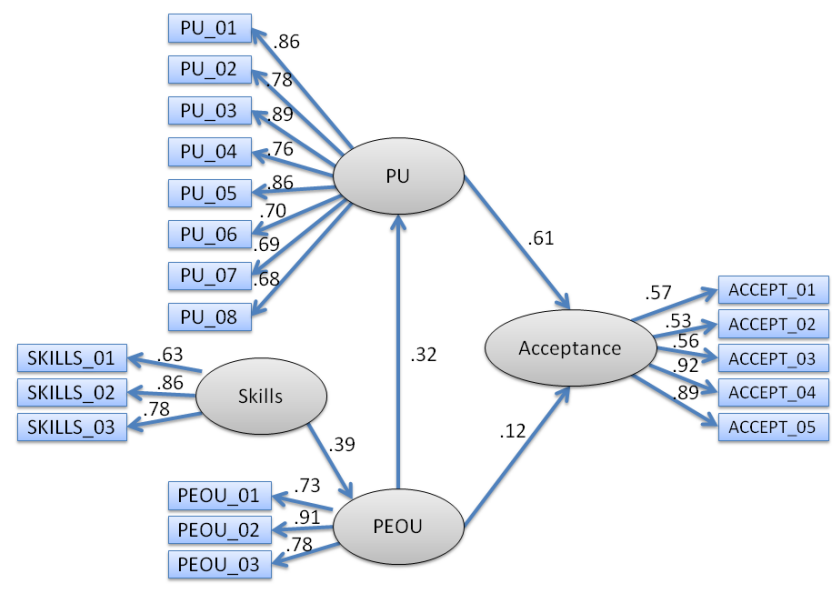

Fig. 4. Standardized estimates for our research model

Two measures that are useful for establishing validity and reliability are the Composite Reliability (CR) and the Average Variance Extracted (AVE). To test for convergent validity we calculated the AVE. For all factors, the AVE was above 0.5 [21] as shown in Table V. To test for discriminant validity we compared the square root of the AVE (on the diagonal in the Table V) to all inter-factor correlations. All factors demonstrated adequate discriminant validity since the diagonal values are greater than the correlations.

We also computed the composite reliability for each factor. In all cases the $\mathrm{CR}$ was above the minimum threshold of 0.70 [21], indicating we have reliability in our factors.

TABLE V

VALIDITY AND RELIABILITY INDICATORS

\begin{tabular}{ccccccc}
\hline & CR & AVE & PEOU & PU & Accep. & Skills \\
\hline PEOU & .850 & .656 & $\mathbf{. 8 1 0}$ & & & \\
PU & .926 & .612 & .320 & $\mathbf{. 7 8 3}$ & & \\
Accep. & .832 & .512 & .314 & .647 & $\mathbf{. 7 1 6}$ & \\
Skills & .803 & .579 & .388 & .080 & .174 & $\mathbf{. 7 6 1}$ \\
\hline
\end{tabular}

Modification indices were consulted to determine if there was an opportunity to improve the model, but there was no need to add any covariance relationship. There are specific measures that are usually computed to determine goodness of fit. Some of these metrics are listed in Table VI, along with their acceptable thresholds according to Hair et al. [21].

For our model, we obtained $\mathrm{cmin} / \mathrm{df}=1.527, \mathrm{CFI}=0.938$, RMSEA $=0.068$ and PCLOSE $=0.058$. These values indicate
TABLE VI

MODEL FIT METRICS AND RECOMMENDED THRESHOLDS, ACCORDING TO HAIR ET AL. [21].

\begin{tabular}{ll}
\hline Metric & Threshold \\
\hline $\begin{array}{l}\text { Chi-square / degrees of freedom } \\
\text { (cmin/df) }\end{array}$ & $<3.000$ good; \\
& $<5$ sometimes permissible \\
\hline & $>0.95$ great; \\
CFI & $>0.90$ traditional; \\
& $>0.80$ sometimes permissible \\
\hline \multirow{2}{*}{ RMSEA } & $<0.05$ good; \\
& 0.05 to 0.10 moderate; \\
\hline PCLOSE & $>0.10 \mathrm{bad}$ \\
\hline
\end{tabular}

that the goodness of fit for our measurement model is acceptable according to the guideline thresholds.

Next, composite variables for factors were created using factor scores in AMOS. After adding the corresponding paths in the model, we performed model fit again. The consulted indicators resulted as follows: $\mathrm{cmin} / \mathrm{df}=1.278, \mathrm{CFI}=0.995$, RMSEA $=0.049$ and PCLOSE $=0.376$. As we can see, the model is within the acceptable range of fitting.

Regression Evaluation of the structure model was performed using AMOS. Table VII shows the estimates resulting for each path. The p-value stands for the degree of significance that the estimate shows the effect on each path, where $* * *$ means that the effects on path is significant in terms of p-value is below 0.001. Thus, the regression weight for (1) Skills in the prediction of Effort, (2) Effort in the prediction of Quality and (3) Quality in the prediction of Acceptance are significantly different from zero at the 0,001 level (two-tailed). On the other hand, the regression weight for Effort in the prediction of Acceptance is only significantly different from zero at the 0.10 level.

TABLE VII

REGRESSION WEIGHTS

\begin{tabular}{ccccc}
\hline Path & Estimate & SE & CR & P \\
\hline PEOU $\leftarrow$ Skills & .477 & .091 & 5.264 & $* * *$ \\
PU $\leftarrow$ PEOU & .415 & .104 & 3.984 & $* * *$ \\
Accep $\leftarrow$ PU & .417 & .046 & 9.096 & $* * *$ \\
Accep $\leftarrow$ PEOU & .093 & .055 & 1.702 & 0.089 \\
\hline
\end{tabular}

\section{Conclusions}

We presented in this work an approach to evaluate the users' acceptance of recommender systems, based on the Technology Acceptance Model. We performed an experiment with a new movie recommender system with real users. Participants answered a post treatment questionary related to a set of variables that influence each latent variable in TAM. Furthermore, we introduced a new latent variable corresponding to believed skills in the use of recommender systems.

A exploratory factor analysis validated the hypothesis that the proposed variables were able to describe adequate, reliable and valid constructs. Then, a confirmatory factor analysis validated the fact that the data fit well in the proposed model. 
Our experiments confirmed that perceived usefulness plays a predominant role for users to accept a new recommender system, as proposed in TAM. Perceived ease of use, on the other hand, did not show to have as much importance as perceived usefulness in the acceptance of the system. However, it did show to have an important role in determining the perceived usefulness itself. Finally, we observed that the skills that the user believes he/she has to use recommender systems have a positive impact on the effort needed to use the new system. These findings would be useful to recommender systems developers both in the academic and commercial areas.

\section{ACKNOWLEGMENT}

This study was partially supported by research projects PIP0181-CONICET and PICT-2011-0366 awarded by ANPCyT, Argentina.

\section{REFERENCES}

[1] F. Ricci, L. Rokach, and B. Shapira, Introduction to Recommender Systems Handbook. Springer, 2011, ch. 1, pp. 1-35.

[2] K. Swearingen and R. Sinha, "Beyond algorithms: An HCI perspective on recommender systems," in ACM SIGIR Workshop on Recommender Systems, vol. 13, 2001, pp. 393-408.

[3] M. Chuttur, "Overview of the technology acceptance model: Origins, developments and future directions," Working Papers on Information Systems, vol. 9, no. 37, pp. 1-22, 2009.

[4] M. G. Armentano, R. Abalde, S. Schiaffino, and A. Amandi, "User acceptance of recommender systems: Influence of the preference elicitation algorithm," in 9th International Workshop on Semantic and Social Media Adaptation and Personalization (SMAP), Nov. 2014, pp. 72-76.

[5] F. D. Davis, "Perceived usefulness, perceived ease of use, and user acceptance of information technology," $M I S Q$, vol. 13, no. 3, pp. 319-340, Sep. 1989.

[6] I. Ajzen and M. Fishbein, Understanding Attitudes and Predicting Social Behavior. Englewood Cliffs, NJ: Prentice-Hall, 1980.

[7] H.-Y. Lee, H. Ahn, and I. Han, "VCR: Virtual community recommender using the technology acceptance model and the user's needs type," Expert Systems with Applications, vol. 33, no. 4, pp. 984-995, Nov. 2007.
[8] A. Asosheh, S. Bagherpour, and N. Yahyapour, "Extended acceptance models for recommender system adaption, case of retail and banking service in iran," WSEAS Trans. on Business and Economics, vol. 5, no. 5, pp. 189-200, May 2008.

[9] R. Hu and P. Pu, "Acceptance issues of personality based recommender systems," in Proc. of ACM RecSys'09. New York, NY, USA: ACM, 2009, pp. 221-224.

[10] D. Baier and E. Stüber, "Acceptance of recommendations to buy in online retailing," Journal of Retailing and Consumer Services, vol. 17, no. 3, pp. 173-180, 2010, new Technologies and Retailing: Trends and Directions. [Online]. Available: http: //www.sciencedirect.com/science/article/pii/S0969698910000202

[11] P. Pu, L. Chen, and R. Hu, "A user-centric evaluation framework for recommender systems," in Proceedings of the fifth ACM conference on Recommender systems. ACM, 2011, pp. 157-164.

[12] H.-C. Chen, C.-C. Hsu, C.-H. Chang, and Y.-M. Huang, "Applying the technology acceptance model to evaluate the learning companion recommendation system on Facebook," in IEEE Fourth International Conference on Technology for Education (T4E), 2012, pp. 160-163.

[13] B. P. Knijnenburg, M. C. Willemsen, Z. Gantner, H. Soncu, and C. Newell, "Explaining the user experience of recommender systems," User Modeling and User-Adapted Interaction, vol. 22, no. 4-5, pp. 441-504, 2012.

[14] Y.-H. Hung, P.-C. Hu, and W.-T. Lee, "Improving the design and adoption of travel websites: An user experience study on travel information recommender systems," in 5th IASDR International Conference, Tokio, Japan, 2013.

[15] V. Venkatesh, M. G. Morris, G. B. Davis, and F. D. Davis, "User acceptance of information technology: Toward a unified view," MIS Quarterly, vol. 27, no. 3, pp. 425-478, 2003.

[16] Y. yao Wang, "Antecedents of review and recommendation systems acceptance," Ph.D. dissertation, Iowa State University, 2011.

[17] P. Pu, L. Chen, and R. Hu, "Evaluating recommender systems from the user's perspective: survey of the state of the art," User Modeling and User-Adapted Interaction, vol. 22, no. 4-5, pp. 317-355, 2012.

[18] H. F. Kaiser, "The varimax criterion for analytic rotation in factor analysis," Psychometrika, vol. 23, no. 3, pp. 187-200, 91958.

[19] — " "The application of electronic computers to factor analysis," Educational and Psychological Measurement, vol. 20, pp. 141-151, 1960.

[20] R. B. Cattell, "The scree test for the number of factors," Multivariate Behavioral Research, vol. 1, pp. 245-276, 1966.

[21] J. F. Hair, W. C. Black, B. J. Babin, and R. E. Anderson, Multivariate data analysis. Prentice Hall Higher Education, 2010. 